PROCEEDINGS OF THE

AMERICAN MATHEMATICAL SOCIETY

Volume 126, Number 11, November 1998, Pages 3191-3198

S 0002-9939(98)04599-7

\title{
ON THE PROJECTIVITY OF MODULE COALGEBRAS
}

\author{
SIU-HUNG NG
}

(Communicated by Ken Goodearl)

\begin{abstract}
In this paper, we derive some criteria for the projectivity of a module coalgebra over a finite dimensional Hopf algebra. In particular, we show that any Hopf algebra over a field of characteristic zero is faithfully flat over its group-like subHopf algebra. Finally, we prove that if $B$ is a finite dimensional subHopf algebra of a Hopf algebra $A$, then $B$ is normal in $A$ if and only if $A B^{+}=B^{+} A$. This improves a result by S. Montgomery (1993).
\end{abstract}

\section{Preliminary}

Let $B$ be a Hopf algebra over the field $k$. For any left $B$-module $M$, let $M^{B}$ denote the space of invariants of $M$, that is

$$
M^{B}=\{x \in M \mid b x=\varepsilon(b) x \text { for } b \in B\},
$$

where $\varepsilon$ is the counit of $B$. If $B$ is finite dimensional, $B^{B}$ is just the space of left integrals $\int_{B}^{l}$. Similarly, we write $\int_{B}^{r}$ for the space of right integrals of $B$. We let ${ }_{M} I$ denote the left $B$-submodule which consists of the elements $x \in M$ such that $\int_{B}^{r} x=0$. Similarly, if $M$ is a right $B$-module, $I_{M}$ denotes the $B$-submodule which annihilates the left integrals of $B$.

For any left $B$-modules $M$ and $N$, the space $\operatorname{Hom}(M, N)$ of $k$-linear homomorphisms admits a natural left $B$-module structure given by

$$
(h \cdot f)(x)=\sum_{(h)} h_{1} f\left(S\left(h_{2}\right) x\right)
$$

for $f \in \operatorname{Hom}(M, N), x \in M, h \in B$, where $S$ is the antipode of $B$. In particular, if $N$ is the trivial $B$-module $k$, the $B$-module action on $M^{*}=\operatorname{Hom}(M, k)$ is given by

$$
(b \neg f)(x)=f(S(b) x)
$$

for $f \in M^{*}, x \in M$ and $b \in B$.

A left (right) $B$-module $C$ is called a left (right) $B$-module coalgebra if $C$ is a coalgebra such that the diagonal map $\Delta_{C}: C \longrightarrow C \otimes C$ and the counit $\varepsilon_{C}: C \longrightarrow k$ are left (right) $B$-module maps, where $k$ is considered as a trivial $B$-module. We write $C^{+}$for the coideal $C \cap \operatorname{ker} \varepsilon_{C}$. We will call $D \subseteq C$ a $B$-submodule coalgebra if $D$ is a subcoalgebra of $C$ and is invariant under the $B$-action. If $X, Y \subseteq C$, recall [8] that the "wedge" $X \wedge Y$ is defined as

$$
X \wedge Y=\Delta_{C}^{-1}(X \otimes C+C \otimes Y) .
$$

Received by the editors September 27, 1996 and, in revised form, April 3, 1997. 1991 Mathematics Subject Classification. Primary 16W30.

(C)1998 American Mathematical Society 
We define $\bigwedge^{1} X=X$ and $\bigwedge^{n+1} X=X \wedge\left(\bigwedge^{n} X\right)$. In particular, if $C_{0} \subseteq X$,

$$
\sum_{n \geq 1} \bigwedge^{n} X=C
$$

where $C_{0}$ is the coradical of $C$ (cf. [4],[8]).

Let $C$ be a $B$-module coalgebra. $M$ is a called a left $(C, B)$-Hopf module if $M$ is a left $C$-comodule and a left $B$-module such that the comodule structure map $\rho_{M}: M \longrightarrow C \otimes M$ is a left $B$-module map. If $C$ is also projective as a $B$-module, we simply call $C$ a projective $B$-module coalgebra. The category ${ }_{B}^{C} \mathcal{M}$ of all left $(C, B)$-Hopf modules is an abelian category.

\section{Projectivity of module CoAlgebra}

Proposition 1. Let $B$ be a finite dimensional Hopf algebra.

(i) If $C$ is a left $B$-module coalgebra, then $C$ is a projective $B$-module if and only if ${ }_{C} I \subseteq \operatorname{ker} \varepsilon_{C}$.

(ii) If $C$ is a right $B$-module coalgebra, then $C$ is a projective $B$-module if and only if $I_{C} \subseteq \operatorname{ker} \varepsilon_{C}$.

Proof. (i) Let us consider the $B$-module $\operatorname{Hom}(C, B)$ of linear homomorphisms from $C$ to $B$. As a $B$-module, $\operatorname{Hom}(C, B)$ is isomorphic to $B \otimes C^{*}$ under the identification

$$
(b \otimes f)(x)=f(x) b
$$

for $f \in C^{*}, x \in C$ and $b \in B$. Let $C_{0}^{*}$ be the trivial $B$-module with the same underlying space as $C^{*}$. It is easy to see that $B \otimes C_{0}^{*}$ is isomorphic to $B \otimes C^{*}$ under the $B$-module isomorphism $\phi: B \otimes C_{0}^{*} \longrightarrow B \otimes C^{*}$ given by

$$
\phi(b \otimes f)=\sum_{(b)} b_{1} \otimes\left(b_{2} \neg f\right)
$$

for $b \in B$ and $f \in C^{*}$. Therefore, $\phi\left(\left(B \otimes C_{0}^{*}\right)^{B}\right)=\left(B \otimes C^{*}\right)^{B}=\operatorname{Hom}_{B}(C, B)$. Since $\left(B \otimes C_{0}^{*}\right)^{B}=\int_{B}^{l} \otimes C^{*}$,

$$
\operatorname{Hom}_{B}(C, B)=\sum_{(\Lambda)} \Lambda_{1} \otimes \Lambda_{2} \rightarrow C^{*} .
$$

where $\Lambda$ is a non-zero left integral of $B$. Let $f \in C^{*}$ and $x \in C$.

$$
\begin{aligned}
\varepsilon_{B} \circ \sum_{(\Lambda)}\left(\Lambda_{1} \otimes \Lambda_{2} \neg f\right)(x) & =\varepsilon_{B}\left(\Lambda_{1}\right) f\left(S\left(\Lambda_{2}\right) x\right) \\
& =f(S(\Lambda) x) .
\end{aligned}
$$

Note that $S(\Lambda)$ is a right non-zero integral of $B$. Therefore, ${ }_{C} I \subseteq$ ker $\varepsilon_{C}$ iff there exists $f \in C^{*}$ such that $f(S(\Lambda) x)=\varepsilon_{C}(x)$ for $x \in C$ which is equivalent to $\varepsilon_{B} \circ \sum_{(\Lambda)}\left(\Lambda_{1} \otimes \Lambda_{2} \rightarrow f\right)=\varepsilon_{C}$. By virtue of Doi's Theorem ([2], Corollary 1), the proof is completed. (ii) can be proved similarly.

A particular case of a result of Takeuchi ([9], Corollary 3.5) is then an immediate consequence of the above proposition.

Corollary 2. Let $B \subseteq A$ be a Hopf algebras, with $B$ finite dimensional. Then $A$ is a left projective $B$-module iff $A$ is a right projective $B$-module. 
Proof. If $A$ is not projective as a left $B$-module, then by Proposition 1 there exists $a \in A$ such that $\int_{B}^{r} a=0$ and $\varepsilon_{A}(a) \neq 0$. Let $S$ be the antipode of $A$. Then $S(a) S\left(\int_{B}^{r}\right)=0$ and $\varepsilon_{A}(S(a))=\varepsilon_{A}(a) \neq 0$. Since $S\left(\int_{B}^{r}\right)=\int_{B}^{l}, A$ is not a right projective $B$-module by Proposition 1 .

\section{Projectivity for Hopf algebras over group-like subalgebras}

Let $A$ be a Hopf algebra and $B$ a subHopf algebra of $A$. Following [9], we use " $B$-projective" to mean "projective $B$-module".

Lemma 3. If the antipode of $B$ is bijective, then the following statements are equivalent :

(a) $A$ is left $B$ faithfully flat;

(b) $A$ is right $B$ faithfully flat;

(c) $A$ is left $B$-projective;

(d) $A$ is right $B$-projective;

(e) $A$ is a left $B$-projective generator;

(f) $A$ is a right B-projective generator;

(g) for any simple subcoalgebra $C$ of $A, B C$ is a projective $B$-module;

(h) if $M \in_{B}^{A} \mathcal{M}$ and $M=B V$ for some simple left $A$-comodule $V$, then $M$ is a projective $B$-module;

(i) for $M \in_{B}^{A} \mathcal{M}, M$ is left $B$-flat.

Proof. By [9], Corollary 3.5, (a) to (f) are all equivalent statements. (e) $\Rightarrow$ (g) and $(\mathrm{g}) \Rightarrow(\mathrm{h})$ are consequences of [2], Theorem 4 .

(h) $\Rightarrow$ (i) Let $M \in_{B}^{A} \mathcal{M}$ and $\mathcal{S}$ be the set of all left $(A, B)$-subcomodules $J$ of $M$ such that $J$ is a flat left $B$-module. The assumption (h) assures that $\mathcal{S} \neq \emptyset$. Since flatness is preserved under direct limit, by Zorn's Lemma there is a maximal element $J_{0} \in \mathcal{S}$. We claim that $J_{0}=M$. If not, there exists a simple left $A$ subcomodule $\bar{V}$ of $M / J_{0}$. Then $B \bar{V}$ is a flat $B$-module. Let $V \supset J_{0}$ be the left $(A, B)$-submodule of $M$ such that $V / J_{0}=B \bar{V}$. Then $V / J_{0}=B \bar{V}$ and we have the exact sequence in ${ }_{B}^{A} \mathcal{M}$

$$
0 \longrightarrow J_{0} \longrightarrow V \longrightarrow B \bar{V} \longrightarrow 0 \text {. }
$$

As flatness is preserved under extension, $V$ is flat and hence $V \in \mathcal{S}$. This contradicts the maximality of $J_{0}$. Therefore $J_{0}=M$, and hence $M$ is left $B$ flat.

(i) $\Rightarrow$ (a) Let $N$ be a non-zero right $B$-module. By applying the functor $N \otimes_{B}$ ? to the exact sequence of left $B$-modules :

$$
0 \longrightarrow B \longrightarrow A \longrightarrow A / B \longrightarrow 0
$$

we have the long exact sequence

$$
\cdots \longrightarrow \operatorname{Tor}_{1}(N, A / B) \longrightarrow N \otimes_{B} B \longrightarrow N \otimes_{B} A \longrightarrow N \otimes_{B}(A / B) \longrightarrow 0 .
$$

By assumption (i), $A$ and $A / B$ are left $B$-flat since they are left $(A, B)$-modules. Therefore $\operatorname{Tor}_{1}(N, A / B)=0$, and so $N \otimes_{B} A \neq 0$. Hence $A$ is left $B$ faithfully flat.

Remark. If $B$ is a Hopf subalgebra of $A$ with bijective antipode, then by virtue of the above lemma, the adjectives "left" and "right" can be dropped. For example, we will simply say $A$ is faithfully flat over $B$ instead of $A$ is left (or right) $B$ faithfully flat. 
Let $G(A)$ denote the set of all group-like elements of $A$. Let $G \subseteq G(A)$ be a subgroup of $G(A)$ and $B=k[G]$.

Lemma 4 ([6], Proposition 2). If $C$ is a simple subcoalgebra of $A$, then

(i) $G_{C}=\{g \in G \mid g C=C\}$ is a finite subgroup of $G$, and

(ii) $B C=\bigoplus_{g \in S} g C$, where $S$ is a set of left coset representatives of $G_{C}$ in $G$.

Proposition 5. The following statements are equivalent :

(i) $A$ is $k[G]$-projective;

(ii) for any subgroup $H$ of $G, A$ is projective over $k[H]$;

(iii) for any finite subgroup $H$ of $G, A$ is projective over $k[H]$.

Proof. (i) $\Rightarrow$ (ii) Suppose $A$ is left $k[G]$-projective. Then $A$ is a direct summand of a free $k[G]$-module $F$. Since $k[G]$ is a free left $k[H]$-module, $F$ is a free left $k[H]$-module. Hence $A$ is left $k[H]$-projective.

(ii) $\Rightarrow$ (iii) is obvious.

(iii) $\Rightarrow$ (i) Assume that $A$ is left $k[H]$-projective for any finite subgroup $H$ of $G$. Let $C$ be a simple subcoalgebra of $A$. By Lemma $4, H=G_{C}$ is a finite subgroup of $G$. Consider the map $\mu: B \otimes_{k[H]} C \longrightarrow B C, \mu: b \otimes c \mapsto b c$. Clearly, $\mu$ is left $B$-linear and surjective. By Lemma 4 (ii), $\mu$ is also injective and hence

$$
B \otimes_{k[H]} C \cong B C
$$

as $B$-modules. By Lemma $3, C$ is a projective $k[H]$-module. Since the functor $B \otimes_{k[H]}$ ? preserves projective objects, $B C$ is $B$-projective. It follows from Lemma 3 that $A$ is $B$-projective.

Theorem 6. Let $A$ be a Hopf algebra over a field $k$ and $G$ be a subgroup of $G(A)$.

(i) If char $k=0, A$ is faithfully flat over $k[G]$.

(ii) If char $k=p>0, A$ is faithfully flat over $k[G]$ if and only if $A$ is projective over $k[H]$ for any finite $p$-subgroup of $G$.

Proof. (i) For any finite subgroup $H$ of $G, k[H]$ is semisimple by Maschke's Theorem. Hence, every left $k[H]$-module is projective and, in particular, $A$ is projective over $k[H]$. By Proposition 5, $A$ is faithfully flat over $k[G]$.

(ii) Let $H$ be a finite subgroup of $G$ and $N$ a $p$-Sylow subgroup of $H$. Notice that every $k[H]$-module $M$ can be embedded into a free $k[H]$-module $F$. If $M$ is $k[N]$-projective, then $M$ is also $k[N]$-injective and so $M$ is a summand of $F$ as $k[N]$-module. Therefore, $M$ is a summand of $F$ as $k[H]$-module (cf. [1], 63.7). Hence, the result follows from Proposition 5.

\section{The existence of a Unique maximal PROJECTIVE MODULE SUBCOALGEBRA}

Proposition 7. Let $B$ be a finite dimensional Hopf algebra and $C$ a left B-module coalgebra.

(i) If $C_{1}$ and $C_{2}$ are projective $B$-submodule coalgebras, then $C_{1} \wedge C_{2}$ and $C_{1}+C_{2}$ are $B$-projective.

(ii) Let $\left\{C_{i}\right\}_{i \in J}$ be a family of projective $B$-submodule coalgebras of $C$. Then $\sum_{i \in J} C_{i}$ is also a projective $B$-module. 
Proof. Since $B$ is a Frobenius algebra, $C_{1}$ is injective as a $B$-module. Let $C_{1}^{\prime}$ be a $B$ submodule of $C$ such that $C=C_{1} \oplus C_{1}^{\prime}$. Then $C_{1} \otimes C+C \otimes C_{2}=C_{1} \otimes C \oplus C_{1}^{\prime} \otimes C_{2}$ as a $B$-module. Let $x \in C_{1} \wedge C_{2}$ such that $\int_{B}^{r} x=0$. Then $\Delta(x)=\sum_{i} a_{i} \otimes b_{i}+\sum_{i} c_{i} \otimes d_{i}$ where $\sum_{i} a_{i} \otimes b_{i} \in C_{1} \otimes C$ and $\sum_{i} c_{i} \otimes d_{i} \in C_{1}^{\prime} \otimes C_{2}$. For $\Lambda \in \int_{B}^{r}, \Delta(\Lambda x)=0$. Therefore,

$$
\sum_{i, j} \Lambda_{j} a_{i} \otimes \Lambda_{j}^{\prime} b_{i}+\sum_{i, j} \Lambda_{j} c_{i} \otimes \Lambda_{j}^{\prime} d_{i}=0
$$

where $\Delta(\Lambda)=\sum_{j} \Lambda_{i} \otimes \Lambda_{i}^{\prime}$. Thus,

$$
\begin{aligned}
& \sum_{i, j} \Lambda_{j} a_{i} \otimes \Lambda_{j}^{\prime} b_{i}=0, \\
& \sum_{i, j} \Lambda_{j} c_{i} \otimes \Lambda_{j}^{\prime} d_{i}=0 .
\end{aligned}
$$

Applying $i d \otimes \varepsilon$ to equation (1) and $\varepsilon \otimes i d$ to equation (2), we have

$$
\begin{aligned}
& \Lambda\left(\sum_{i} \varepsilon\left(b_{i}\right) a_{i}\right)=0, \\
& \Lambda\left(\sum_{i} \varepsilon\left(c_{i}\right) d_{i}\right)=0 .
\end{aligned}
$$

By Proposition 1, $\varepsilon\left(\sum_{i} \varepsilon\left(b_{i}\right) a_{i}\right)=0$ and $\varepsilon\left(\sum_{i} \varepsilon\left(c_{i}\right) d_{i}\right)=0$. Thus, $\varepsilon(x)=0$. Since $C_{1} \wedge C_{2}$ is obviously a $B$-submodule coalgebra, $C_{1} \wedge C_{2}$ is projective by Proposition 1. Obviously, $C_{1}, C_{2}$ are left $\left(C_{1} \wedge C_{2}, B\right)$-Hopf modules and so is $C_{1}+C_{2}$. By [2](Theorem 4), $C_{1}+C_{2}$ is a projective $B$-module.

(ii) Let $x \in \sum_{i \in I} C_{i}$ such that $\Lambda x=0$. Then there exist $C_{i_{1}}, \cdots, C_{i_{n}}$ such that $x \in \sum_{k=1}^{n} C_{i_{k}}$. By (i), $\sum_{k=1}^{n} C_{i_{k}}$ is projective and so $\varepsilon(x)=0$ by Proposition 1 . Hence $\sum_{i \in I} C_{i}$ is $B$-projective by Proposition 1.

Corollary 8. Let $B$ be a finite dimensional Hopf algebra ${ }^{1}$. For any left B-module coalgebra $C$, there exists a unique maximal projective $B$-submodule coalgebra $P(C)$. Moreover, $P(C)$ is co-idempotent, i.e. $P(C) \wedge P(C)=P(C)$.

Proof. Let $\mathcal{S}$ be the set of all projective $B$-submodule coalgebras of $C$. By Proposition 7, $P(C)=\sum_{D \in \mathcal{S}} D$ is then the largest projective $B$-submodule coalgebra of $C . P(C) \wedge P(C)=P(C)$ is a direct consequence of Proposition 7 (i) and the maximality of $P(C)$.

Corollary 9. Let $B$ be a finite dimensional Hopf algebra and $C$ a B-module coalgebra. (i) If $D$ is a B-submodule coalgebra of $C, P(D)=P(C) \cap D$. (ii) If $C$ is a direct sum of $B$-submodule coalgebras $\left\{C_{i}\right\}$, then $P(C)=\bigoplus_{i} P\left(C_{i}\right)$.

Proof. (i) By Corollary $8, P(D) \subseteq P(C)$ and hence $P(D) \subseteq P(C) \cap D$. Conversely, by Theorem 4 of [2], $P(C) \cap D$ is a projective $B$-module. Then, we have $P(C) \cap D \subseteq$ $P(D)$.

(ii) If $C=\bigoplus_{i} C_{i}$ as $B$-module coalgebra, it follows by Theorem 3 of [3] that $P(C)=\bigoplus_{i}\left(P(C) \cap C_{i}\right)$. Hence, by (i), $P(C)=\bigoplus_{i} P\left(C_{i}\right)$.

Corollary 10. Let $B$ be a finite dimensional Hopf algebra and $C$ a B-module coalgebra. The following statements are equivalent:

${ }^{1}$ After this paper was written, the author was able to eliminate the finite dimension hypothesis on $B$ using different techniques. 
(i) $C$ is a projective $B$-module,

(ii) $B C_{0}$ is $B$-projective, where $C_{0}$ is the coradical of $C$,

(iii) $B D$ is $B$-projective for any simple subcoalgebra $D$ of $C$.

Proof. (i) $\Rightarrow$ (iii) is a direct consequence of Theorem 4 in [2].

(iii) $\Rightarrow$ (ii) follows from Proposition 7 (ii).

(ii) $\Rightarrow$ (i) Since $B C_{0}$ is $B$-projective, by Proposition 7 (i), $\bigwedge^{n} B C_{0}$ is $B$-projective for any $n \geq 1$. Hence, by Proposition 7 (ii), $\sum_{n \geq 1} \wedge^{n} B C_{0}$ is $B$-projective. The result follows from the fact that $C=\sum_{n \geq 1} \wedge^{n} B \bar{C}_{0}$.

\section{Normal SubHopf Algebras}

Definition 11. Let $A$ be any Hopf algebra, $B$ a subHopf algebra of $A$ and $S$ the antipode of $A$.

(1) $B$ is left normal if

$$
a \triangleright b=\sum a_{1} b S\left(a_{2}\right) \in B
$$

for $a \in A$ and $b \in B$.

(2) $B$ is right normal if

$$
b \triangleleft a=\sum S\left(a_{1}\right) b a_{2} \in B
$$

for $a \in A$ and $b \in B$.

(3) $B$ is normal if $B$ is left normal and right normal.

It is well known that if $B$ is a normal subHopf algebra of $A$, then $A B^{+}=B^{+} A$ (cf. [4], 3.4.2). However, the converse is open. If $A$ is left or right faithfully flat over $B$, the converse is known to be true (cf. [4], 3.4.3 and [9], 4.4). In this section, we will show that the converse statement holds if $B$ is finite dimensional which enhances the result in [4], 3.4.4.

Lemma 12. Let $B$ be a finite dimensional Hopf algebra and $C$ a left B-module coalgebra. Let $\eta_{C}: C \longrightarrow C /\left(B^{+} C\right)$ be the canonical B-module coalgebra homomorphism. Then, $\eta_{C}\left({ }_{C} I\right)$ is a subcoalgebra of $C /\left(B^{+} C\right)$. In particular, if $B$ is a subHopf algebra of a Hopf algebra $A$, then $\eta_{A}\left({ }_{A} I\right)$ is a right $A$-submodule coalgebra of $\eta_{A}(A)$.

Proof. To simplify, we write $\bar{C}$ for $C /\left(B^{+} C\right)$. Clearly, $\bar{C}$ is a left $B$-module coalgebra and $C$ admits a natural left and right $\bar{C}$-comodule structure. Let $\Lambda$ be a nonzero element in $\int_{B}^{r}$. Consider the map $f_{C}: \bar{C} \longrightarrow C$ defined by

$$
f_{C}\left(\eta_{C}(x)\right)=\Lambda x
$$

for $x \in C$ (see [7], p3348). Clearly, the map is well-defined and is a left and right $\bar{C}$-comodule map. Therefore, $\operatorname{ker} f_{C}$ is a subcoalgebra of $\bar{C}$. Notice that ker $f_{C}=\eta_{C}\left(C_{C} I\right)$ and hence the result follows. If $B$ is a subHopf algebra of $A$, then $\eta_{A}$ is a right $A$-module map. Since ${ }_{A} I$ is a right $A$-submodule of $A, \eta_{A}\left({ }_{A} I\right)$ is a right $A$-submodule of $\eta_{A}(A)$.

Corollary 13. Let $B$ be a finite dimensional Hopf algebra and $C$ a left B-module coalgebra. If $C$ is a projective $B$-module, $C_{C} I=B^{+} C$.

Proof. Clearly, $B^{+} C \subseteq C_{C} I$. It suffices to show that ${ }_{C} I \subseteq B^{+} C$. By Proposition $1,{ }_{C} I \subseteq \operatorname{ker} \varepsilon_{C}$. Therefore, $\eta_{C}\left({ }_{C} I\right) \subseteq \operatorname{ker} \varepsilon_{\bar{C}}$. By Lemma $12 \eta_{C}\left({ }_{C} I\right)$ is also a subcoalgebra of $\bar{C}$. Therefore, $\eta_{C}\left({ }_{C} I\right)=0$ and so ${ }_{C} I \subseteq B^{+} C$. 
Lemma 14. Let $A$ be a Hopf algebra and $B$ a finite dimensional subHopf algebra of $A$. If $A$ is not a left projective $B$-module, then

$$
{ }_{A} I I_{A}+A B^{+} A=A \text {. }
$$

Proof. By Lemma 12, ${ }_{A} I /\left(B^{+} A\right)$ is a right $A$-submodule coalgebra of $A /\left(B^{+} A\right)$. Note that $A B^{+} A$ is a Hopf ideal of $A$. Thus $A B^{+} A /\left(B^{+} A\right)$ is also a coideal of $A / B^{+} A$. Therefore, ${ }_{A} \bar{I}=\left({ }_{A} I+A B^{+} A\right) / A B^{+} A$ is a right $A$-submodule coalgebra of $A / A B^{+} A$. In particular, ${ }_{A} \bar{I}$ is a right ideal of $A / A B^{+} A$. Since $A$ is not a projective $B$-module, ${ }_{A} I \nsubseteq A^{+}$by Proposition 1 . Hence ${ }_{A} I \nsubseteq A B^{+} A$, and so ${ }_{A} \bar{I}$ is a non-zero right ideal as well as coideal of $A / A B^{+} A$. Therefore, ${ }_{A} \bar{I}=A / A B^{+} A$ (see [8], p. 108, Exercise 5). Thus, we have

$$
{ }_{A} I+A B^{+} A=A .
$$

By Corollary 2, $A$ is not a projective right $B$-module. By virtue of Proposition 1 (ii), we similarly obtain

$$
I_{A}+A B^{+} A=A .
$$

Hence, we have ${ }_{A} I I_{A}+A B^{+} A=A$.

Theorem 15. Let $A$ be a Hopf algebra and $B$ a finite dimensional subHopf algebra of $A$. If $A B^{+} \subseteq B^{+} A$ or $B^{+} A \subseteq A B^{+}$, then $A$ is a free left (right) $B$-module.

Proof. By the Nichols-Zoeller Theorem [5], it suffices to consider the case when $A$ is infinite dimensional. By Schneider's Theorem ([7], Theorem 2.4), it suffices to show that $A$ is a left projective $B$-module. Suppose $A$ is not a projective left $B$-module. By Lemma 14, we have

$$
{ }_{A} I I_{A}+A B^{+} A=A .
$$

If $A B^{+} \subseteq B^{+} A$, then $A B^{+} A=B^{+} A$. Thus, ${ }_{A} I I_{A}+B^{+} A=A$ and so $\int_{B}^{r} A=0$, contradiction! Similarly, if $B^{+} A \subseteq A B^{+}$, then ${ }_{A} I I_{A}+A B^{+}=A$ and hence $A \int_{B}^{l}=0$, contradiction! Therefore, $A$ is a projective $B$-module.

Corollary 16. Let $A$ be a Hopf algebra and $B$ a finite dimensional subHopf algebra of $A$. Then

(i) $B$ is left normal iff $A B^{+} \subseteq B^{+} A$;

(ii) $B$ is right normal iff $B^{+} A \subseteq A B^{+}$;

(iii) $B$ is normal iff $B^{+} A=A B^{+}$.

(iv) If the antipode of $A$ is bijective, $B$ is left normal iff $B$ is right normal.

Proof. (i) If $B$ is left normal, it is obvious that $A B^{+} \subseteq B^{+} A$ (see [9], 1.4). Conversely, assume $A B^{+} \subseteq B^{+} A$. By Theorem $15, A$ is a free right $B$-module and hence faithfully flat. By Theorem 4.4 of $[9], B$ is left normal. Similarly, we can prove (ii).

(iii) An immediate consequence of (i) and (ii).

(iv) A direct consequence of Theorem 15 and Corollary 4.5 of [9].

\section{ACKNOWLEDGMENT}

I would like to express my appreciation to Professors E. Taft and C. Weibel for their helpful suggestions and encouragement while writing this paper. I also wish to thank the referee for his or her valuable comments. 


\section{REFERENCES}

[1] C. W. Curtis and I. Reiner, Representation Theory of Finite Groups and Associative Algebras (Interscience, New York, 1962). MR 90g:16001

[2] Y. Doi, On the structure of relative Hopf modules, Comm. Algebra 11 (1983), 243-255. MR 84a:16014

[3] I. Kaplansky, Bialgebras, Chicago Press, 1975. MR 55:8087

[4] S. Montgomery, Hopf algebras and their actions on rings, CBMS Lecture Notes, vol. 82, Amer. Math. Soc., Providence, RI, 1993. MR 94i:16019

[5] W. D. Nichols and M. B. Zoeller, A Hopf algebra freeness theorem, Amer. J. Math. 111 (1989), 381-385. MR 90c:16008

[6] D. E. Radford, Freeness (projectivity) criteria for Hopf algebras over Hopf subalgebras, J. Pure Appl. Algebra 11 (1977) 15-28. MR 57:16344

[7] H.-J. Schneider, Some remarks on exact sequences of quantum groups. Comm. Algebra 21 (1993), 3337-3357. MR 94e:17026

[8] M. Sweedler, Hopf algebras, Benjamin, New York, 1969. MR 40:5705

[9] M. Takeuchi, Quotient spaces for Hopf algebras. Comm. Algebra 22 (1994), 2503-2523. MR 95h:16055

Department of Mathematics, Rutgers University, New Brunswick, New Jersey 08903 Current address: Department of Mathematics, University of California at Santa Cruz, Santa Cruz, California 95064

E-mail address: shng@math.ucsc.edu 